\title{
Experimental Determination of Optimum Coil Pitch for a Planar Mesh-Type Micromagnetic Sensor
}

\author{
Subhas C. Mukhopadhyay, Senior Member, IEEE, Sotoshi Yamada, Member, IEEE, and \\ Masayoshi Iwahara, Member, IEEE
}

\begin{abstract}
To overcome the directional properties of a planar meander-type sensor, a new planar micromagetic sensor having mesh-type configuration is reported in this paper. Analytical models are usually used for the characterization of the planar-type sensors. Sensors having mesh-type configuration have been fabricated for the derivation of the optimum coil pitch.
\end{abstract}

Index Terms-Meander-type configuration, mesh-type configuration, near-surface material properties, nondestructive evaluation techniques, planar-type sensors.

\section{INTRODUCTION}

$\mathbf{F}$ OR THE estimation of the near-surface material properties (e.g., conductivity, permeability, thickness, etc.), the application of a planar-type meander magnetic sensor has already been reported [1], [2]. In order to overcome the directional properties of meander-type sensors, a new type of sensor having a mesh configuration has been fabricated. A two-dimensional (2-D) analytical model of one pitch of the sensor has been developed for the calculation of transfer impedance and other parameters. The problem of edge effect is difficult to overcome in the analytical model. This paper reports the selection of the optimum pitch based on the experimental results. Five types of sensor having pitches of $1.4,1.8,2.166,2.6$, and $3.25 \mathrm{~mm}$ have been fabricated.

The transfer impedance (i.e., the ratio of voltage of the sensing coil to the current of the exciting coil) is used to evaluate the near-surface material properties which can predict the mechanical fatigue and some other characteristics of the material [3]. A neural network model is used for the postprocessing of the parameters from the measured impedance data.

\section{CONFiguration OF SENSOR}

The sensor consists of two coils. The exciting coil carries a high-frequency sinusoidal current to generate the ac magnetic field. The sensing coil is placed above the exciting coil and is used to pick up the induced voltage. Fig. 1 shows the configuration of a planar meander-type magnetic sensor fabricated and developed in the laboratory for the inspection of defects of the printed circuit board (PCB) [4]. The conductors in the PCB

Manuscript received January 31, 2002; revised May 14, 2002

S. C. Mukhopadhyay is with the Institute of Information Sciences and Technology, Massey University, Palmerston North, New Zealand (e-mail: S.C.Mukhopadhyay@massey.ac.nz).

S. Yamada and M. Iwahara are with the Faculty of Engineering, Kanazawa University, Kanazawa 920, Japan (e-mail: yamada@magstar.ec.t.kanazawa-u. ac.jp; iwahara@magstar.ec.t.kanazawa-u.ac.jp).

Digital Object Identifier 10.1109/TMAG.2002.802285.

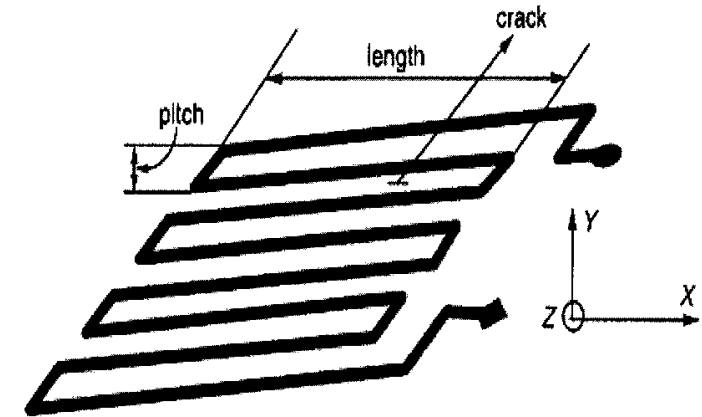

Fig. 1. Configuration of meander-type magnetic sensor.

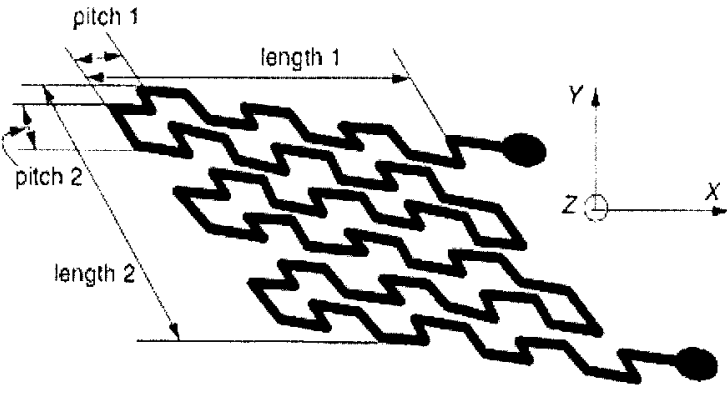

Fig. 2. Configuration of mesh-type magnetic sensor

being long, the meander-type configuration is the only option available, but it is difficult to get any information about the inner layer crack aligned in parallel with the meander conductor as shown in Fig. 1. Since the detection of the existence of cracks is based on the effect of eddy-current flow, a crack parallel to the exciting meander conductor will affect the flow of eddy currents only slightly. In order to overcome the directional property, a planar mesh-type micromagnetic sensor as shown in Fig. 2 is developed and is used for the estimation of near-surface material properties.

\section{EXPERIMENTAL DETERMINATION OF OPTIMUM COIL PITCH}

Usually, an analytical model is used for the characterization of the sensor and one coil pitch is assumed for that purpose, as shown in Fig. 3. This is based on the assumption that the length of the coil is very large compared to the pitch so that the edge effect can be neglected. For the meander-type configuration, the length is more than 10 times the pitch, therefore, the error due to the edge effect is small. But in the mesh-type configuration, because the length (e.g., pitch 1 in Fig. 2) is the same as the length of the pitch (pitch 2), the edge effect cannot be neglected. This effect is clearly seen in the analytical results. A typical 


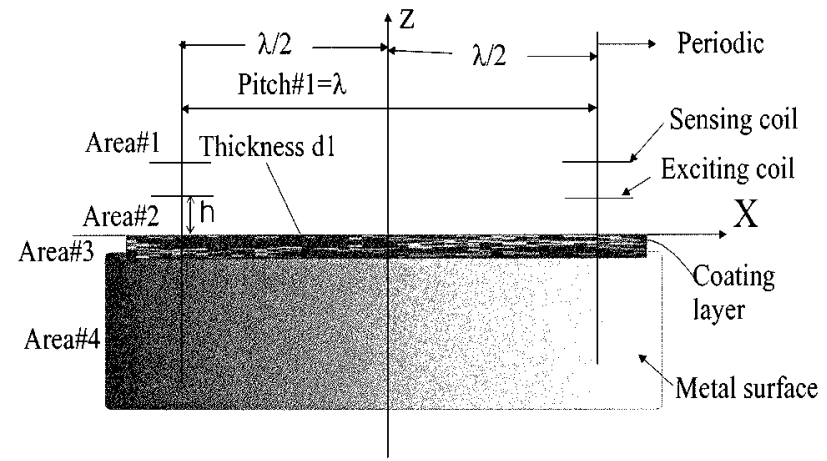

Fig. 3. Two-dimensional representation of analytical model of one coil pitch.

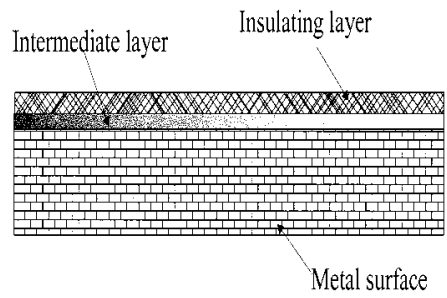

Fig. 4. Schematic of electroplated material.

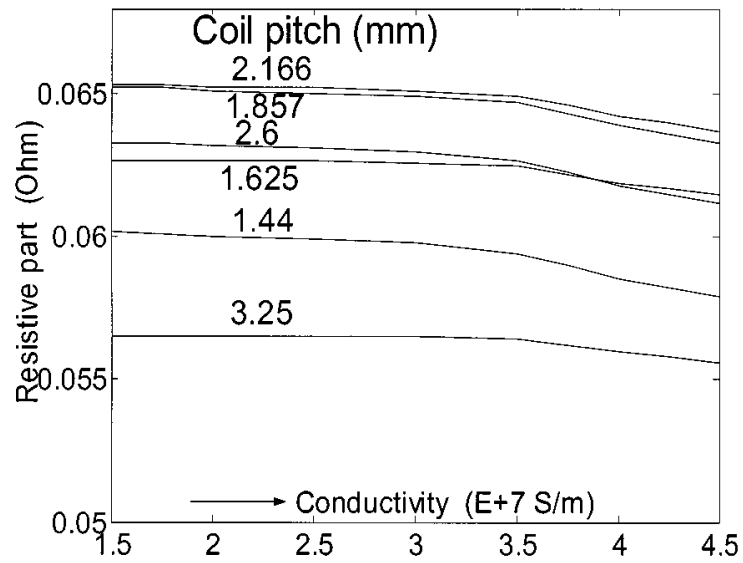

Fig. 5. Variation of resistive part of the transfer impedance with the conductivity of the intermediate layer for different coil pitches.

case is presented here. To inspect the electroplated material used in the blade in a gas turbine, catastrophic failure is very much dependent on the intermediate layer, as shown in Fig. 4.

A few analytical results for this case are presented here. Fig. 5 shows the variation of the resistive part with the conductivity of the intermediate layer for different coil pitches. It is seen that the resistive part is a maximum corresponding to the coil pitch of $2.166 \mathrm{~mm}$. Fig. 6 shows the variation of the resistive part with the liftoff of the sensor for different coil pitches. It is very difficult to choose the optimum pitch of the sensor from this result. In order to avoid this problem, an experimental approach is adopted for the determination of optimum coil pitch of the sensor.

Five types of sensor, having pitches of 1.4, 1.8, 2.166, 2.6, and $3.25 \mathrm{~mm}$, have been fabricated and characterized using the experimental setup as shown in Fig. 7. A Hewlett-Packard impedance analyzer HP 4194A is used for the measurement of transfer impedance. A square coil pitch has been assumed here,

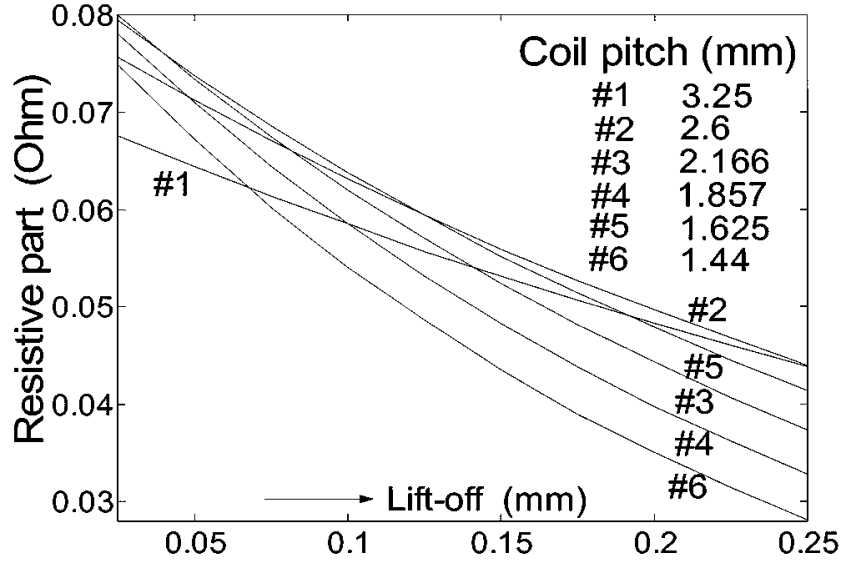

Fig. 6. Variation of resistive part of the transfer impedance with the liftoff of the sensor for different coil pitches.

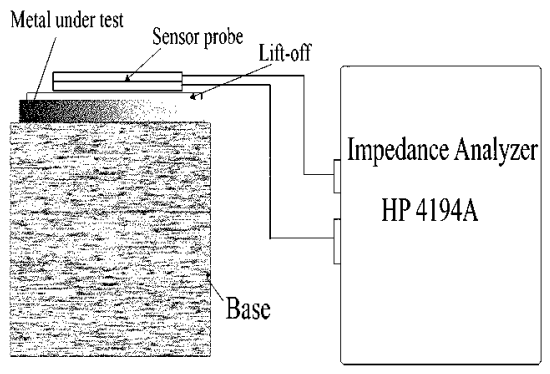

Fig. 7. Experimental setup.

TABLE I

EXPERIMENTAL DATA

\begin{tabular}{|c|c|c|c|c|c|}
\hline Material & $\begin{array}{c}\text { Pitch } \\
1.4 \mathrm{~mm}\end{array}$ & \begin{tabular}{|c|} 
Pitch \\
$1.8 \mathrm{~mm}$
\end{tabular} \mid & $\begin{array}{c}\text { Pitch } \\
2.166 \mathrm{~mm}\end{array}$ & $\begin{array}{c}\text { Pitch } \\
2.6 \mathrm{~mm}\end{array}$ & $\begin{array}{l}\text { Pitch } \\
3.25 \mathrm{~mm}\end{array}$ \\
\hline Copper & 0.0559 & 0.0639 & 0.0642 & 0.0623 & 0.0589 \\
\hline Aluminum & 0.0748 & 0.0862 & 0.0875 & 0.0839 & 0.0786 \\
\hline $\begin{array}{c}\text { Molybde } \\
\text { Num }\end{array}$ & 0.0913 & 0.1112 & 0.1159 & 0.1096 & 0.1054 \\
\hline ngs & 0.0915 & 0.1124 & 0.1167 & 0.1103 & 0.1079 \\
\hline
\end{tabular}

which means pitch 1 and pitch 2 of Fig. 2 are the same. An integer number of coil pitches has been assumed and the area covered by each of them is kept constant.

Table I shows the experimental results. The resistive part of the transfer impedance for different surface materials with different coil pitches of the sensor are shown. It is seen that the resistive part of the impedance increases with the increase in coil pitch from 1.4 to $2.166 \mathrm{~mm}$ and then starts decreasing. So the coil pitch corresponding to $2.166 \mathrm{~mm}$ is chosen as the optimum coil pitch for the present configuration of the sensor. The impedance is used for the estimation of surface material properties through a neural network model.

\section{EXPERIMENTAL IMPEDANCE DATA AND ESTIMATION OF NEAR-SURFACE MATERIAL PROPERTIES}

The transfer impedance of the sensor obtained from the experiment is used for the estimation of near-surface material 


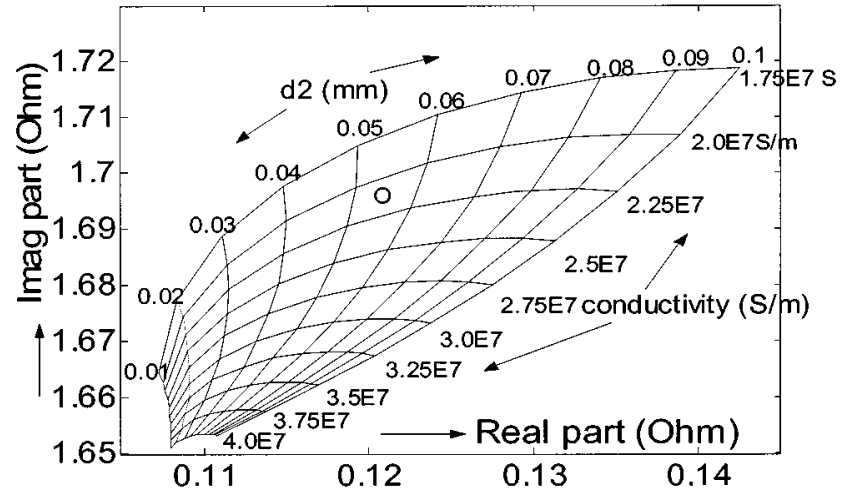

Fig. 8. Grid system for the estimation of surface properties.

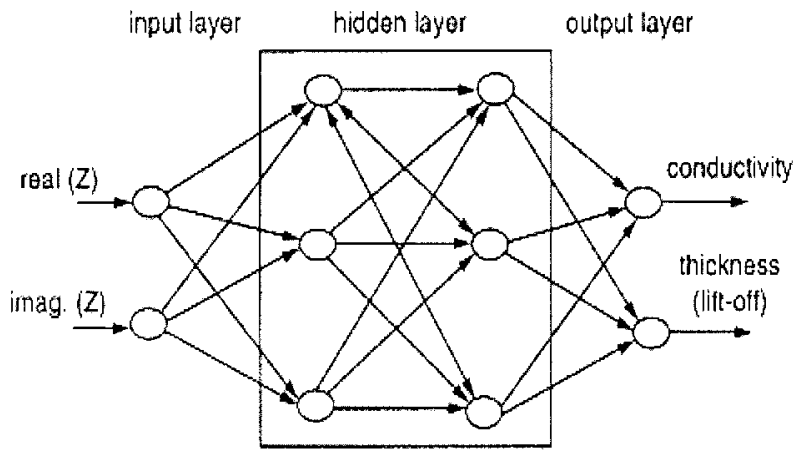

Fig. 9. Schematic representation of neural network model.

properties. The transfer impedance of the sensor depends in a complex way on near-surface material properties along with many other parameters. There is no simple and direct method of estimating the near-surface properties from the impedance data. An approach based on an off-line generated grid system is used in [1]. The data for the grid system is generated from the analytical model and is developed by plotting the imaginary part of the impedance versus its real part for the parameters of interest at one particular frequency. A conductivity-thickness measurement grid, shown in Fig. 8, corresponds to an operating frequency of $1 \mathrm{MHz}$. Each node of the grid system in Fig. 8 has fixed conductivity and thickness. The measured impedance data is plotted on the grid as shown by $\mathbf{O}$ in Fig. 8 and the surface properties are estimated by interpolation technique.

Instead of using the grid system, the use of a neural network model is reported here. A schematic representation of a simple neural network model is shown in Fig. 9 in which the real and imaginary part of the impedance are used as inputs. The conductivity and thickness (or liftoff) are considered as outputs.
TABLE II

COMPARISON OF RESULTS OBTAINED USING GRID SySTEM AND NEURAL NETWORK

\begin{tabular}{lll}
\hline $\begin{array}{l}\text { Conductivity of } \\
\text { aluminium }\end{array}$ & $\begin{array}{l}\text { From grid } \\
\text { system, Sim } \times 10^{7}\end{array}$ & $\begin{array}{l}\text { Using neural } \\
\text { network mode, } \\
S / \mathrm{m} \times 10^{7}\end{array}$ \\
\hline with no lift-off & 3.6 & 3.53 \\
with $0.1 \mathrm{~mm}$ lift-off & 3.7 & 3.54 \\
with $0.2 \mathrm{~mm}$ lift-off & 3.8 & 3.53 \\
with $0.3 \mathrm{~mm}$ lift-off & 3.8 & 3.51 \\
with $0.4 \mathrm{~mm}$ lift-off & 3.4 & 3.49 \\
\hline
\end{tabular}

MATLAB's neural network toolbox has been used for the solution. The data used for the generation of the grid system can be used to train the neural network. Depending on the situation, the model can have two, three, or even more outputs. A simple model has been developed and the results obtained from it are compared with that obtained utilizing a grid system [2] in Table II. It is seen that the level of error is consistent. The level of the error can be reduced using experimental data to train the network.

\section{CONCLUSION}

A new planar micromagnetic sensor having mesh-type configuration is reported in this paper. The optimum pitch of the sensor has been derived from the experimental results. The measured transfer impedance of the sensor has been used for the estimation of near-surface material properties through a simple neural network model.

\section{REFERENCES}

[1] N. J. Goldfine and D. Clark, "Near-surface material property profiling for determination of SCC susceptibility," in Proc. 4th EPRI Balance-ofPlant Heat Exchanger NDE Symp., June 1996, pp. 1-11.

[2] S. C. Mukhopadhyay, S. Yamada, and M. Iwahara, "Investigation of near-surface material properties using planar-type meander coil," in Proc. 10th Int. Symp. Applied Electromagnetics and Mechanics (JSAEM Studies in Applied Electromagnetics and Mechanics), vol. 11, Tokyo, Japan, May 2001, pp. 61-69.

[3] Y. Shi and D. C. Jiles, "Finite element analysis of the influence of a fatigue crack on magnetic properties of steel," J. Appl. Phys., vol. 83, no. 11, pp. 6353-6355, June 1998.

[4] S. Yamada, H. Fujiki, M. Iwahara, S. C. Mukhopadhyay, and F. P. Dawson, "Investigation of printed wiring board testing by using planar coil-type ECT probe," IEEE Trans. Magn., vol. 33, pp. 3376-3378, Sept. 1997. 


\section{Experimental determination of optimum}

coil pitch for a planar mesh-type

\section{micromagnetic sensor}

Mukhopadhyay, S. C.

2002-09

http://hdl.handle.net/10179/9601

22/04/2023 - Downloaded from MASSEY RESEARCH ONLINE 